\title{
Ho:Tm:Er:LuAG AND TWO WAVELENGTH OSCILLATION
}

\author{
Norman P. Barnes \\ Keith E. Murray \\ NASA Langley Research Center \\ Hampton, VA 23681 \\ Brian M. Walsh \\ Boston College \\ Chestnut Hill, MA 02167 \\ Ralph L. Hutcheson \\ Scientific Materials Corporation \\ Bozeman, MT 59715
}

\begin{abstract}
Ho:Tm:Er:LuAG demonstrated simultaneous laser oscillation associated with two different transitions and different atoms. Two wavelength oscillation, or TWO, can be achieved in a single laser and single resonator. Moreover, the wavelength can be electronically controlled by varying the pump pulse length, Either the short wavelength, the $\mathrm{Ho}^{5} \mathrm{I}_{7}$ to ${ }^{5} \mathrm{I}_{8}$ transition at $2.1 \mu \mathrm{m}$, or the long wavelength, the $\mathrm{Er}{ }^{4} \mathrm{I}_{11 / 2}$ to ${ }^{4} \mathrm{I}_{13 / 2}$ transition at $2.7 \mu \mathrm{m}$, or both wavelengths simultaneously can be produced without resorting to mechanical intervention. Consequently, a single laser system can easily switch between producing either of these useful wavelengths. Laser operation of both transitions has been characterized and is presented.
\end{abstract}

\section{Key Words}

Rare earth and transition metal solid state lasers, Laser materials, Rare earth doped materials.

Ho:Tm:Er:LuAG is capable of simultaneous operation on two different wavelengths which are associated with two different transitions and two different atoms, Ho and Er. Ho lases on the ${ }^{5} \mathrm{I}_{7}$ to ${ }^{5} \mathrm{I}_{8}$ transition at $2.1 \mu \mathrm{m}$ while Er lases on the ${ }^{4} I_{11 / 2}$ to ${ }^{4} I_{13 / 2}$ transition at $2.7 \mu \mathrm{m}$. Moreover, laser operation at either or both of these wavelengths can be easily varied by changing the length of the pump pulse. Therefore two wavelength oscillation, or TWO, is controlled electronically and is available from a single laser. The ability to easily switch between the two wavelengths is of particular interest in situations where both wavelengths are useful.
Two wavelength operation of the laser is characterized and controlled by the different pump powers and pump energies. An experimental arrangement for these tests is shown in Fig. 1. Although the system was not optimized for operation on both wavelengths, simultaneous lasing could be obtained. For these experiments, the laser rod was $4.0 \mathrm{~mm}$ in diameter and $80 \mathrm{~mm}$ in length. Unfortunately, the available laser cavity could only pump $67 \mathrm{~mm}$ of the length of the laser rod. Since the $\mathrm{Ho}^{5} \mathrm{I}_{7}$ to ${ }^{5} \mathrm{I}_{8}$ transition is a quasi four level laser, the unpumped length of the laser rod detracts seriously from the efficiency by raising the threshold. In addition, only a few mirrors were available for the experiments. Since the $2.7 \mu \mathrm{m}$ radiation must pass through the output mirror for the $2.1 \mu \mathrm{m}$ transition, a substantial amount of this radiation is lost by being reflected by the $2.1 \mu \mathrm{m}$ mirror, decreasing the slope efficiency. On the other hand, the $2.1 \mu \mathrm{m}$ resonator contains the $2.7 \mu \mathrm{m}$ resonator. Consequently, the $2.1 \mu \mathrm{m}$ radiation must pass through both the high reflector and the output mirror for the $2.7 \mu \mathrm{m}$ radiation, raising the threshold and decreasing the slope efficiency.

\section{Experimental Arrangement}

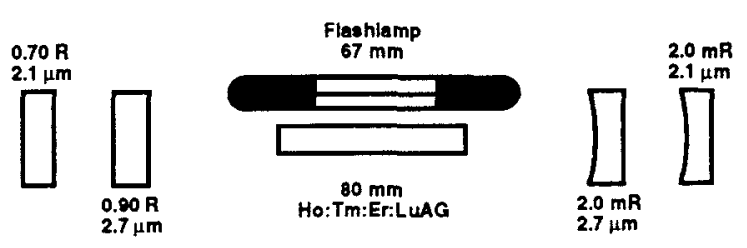

Figure 1. Experimental arrangement for two wavelength operation. 
These problems could be obviated by having mirrors coated especially for two wavelength operation.

Laser output at the two wavelengths occur at different times in the pump pulse. A typical trace of the pump and laser output pulses as a function of time are shown in Fig. 2. Operation of the $\mathrm{Er}^{4} \mathrm{I}_{11 / 2}$ to ${ }^{4} \mathrm{I}_{13 / 2}$ transition occurs first, after about $100 \mu \mathrm{sec}$. Operation on this transition ceases after about $200 \mu \mathrm{sec}$ while operation on the $\mathrm{Ho}^{5} \mathrm{I}_{7}$ to ${ }^{5} \mathrm{I}_{8}$ transition begins after about $350 \mu \mathrm{sec}$. Obviously, for the particular pump power and energy shown in the figure, operation on both wavelengths can be obtained. To obtain operation on only the Er transition, a pump pulse can be electronically terminated after about $200 \mu \mathrm{sec}$ at the pump power shown in the figure. Conversely, to obtain operation on only the Ho transition, a lower pump power can be used. Since the lifetime of the $\mathrm{Ho}^{5} \mathrm{I}_{7}$ is so long, it can integrate the pump energy and lase at a pump power level ${ }_{4}$ is low enough to prevent the $E r{ }^{4} I_{11 / 2}$ to ${ }^{4} I_{13 / 2}$ transition to come to threshold.

Both lasing wavelengths and lifetimes were measured in order to characterize two wavelength operation. Wavelength of the Er transition is measured under laser operation conditions to be $2.699 \mu \mathrm{m}$, close to the peak absorption wavelength of water. Wavelength of the Ho transition depends on mirror reflectivity and is measured under laser operation to be 2.100 or $2.124 \mu \mathrm{m}$, depending on the output mirror reflectivity. Lasing on the shorter wavelength occurs for the lower output mirror reflectivities, indicating a high gain for this transition. Lifetime of the Ho ${ }^{5} \mathrm{I}_{7}$ manifold is dependent on the level of pumping. In this sample, the measured lifetime when extrapolated to low levels of pumping is $7.6 \mathrm{msec}$.

Flashlamp Current and Laser Output

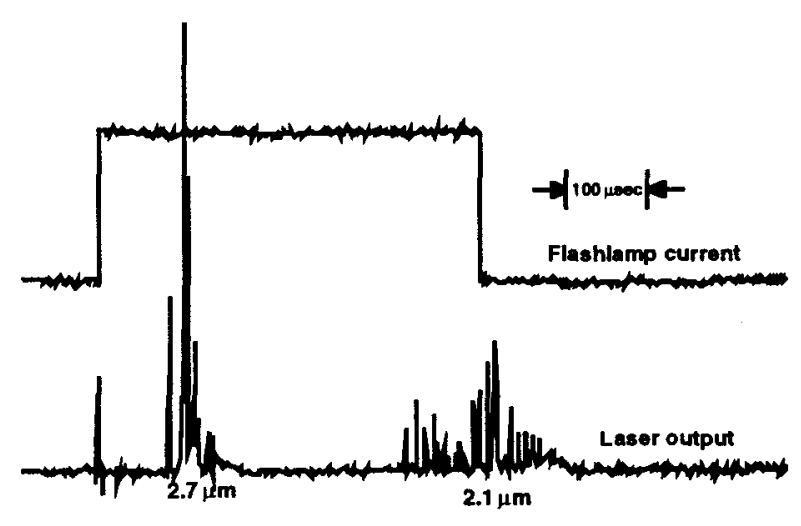

Figure 2. Laser power and flashlamp current versus time. Wavelength of the different pulses are indicated.
Performance of the laser system when operating only on the $\mathrm{Er}^{4} \mathrm{I}_{11 / 2}$ to ${ }^{4} \mathrm{I}_{13 / 2}$ transition is dependent on the pump pulse length. Using a $2.0 \mathrm{~m}$ radius of curvature high reflecting mirror and a 0.90 reflecting flat output mirror, the laser output energy was measured as a function of the electrical energy for three different pump pulse lengths. Laser performance is summarized in Fig. 3. As can be seen, the threshold increases monotonically with pump pulse length, increasing from $21.2 \mathrm{~J}$ to $60.5 \mathrm{~J}$. On the other hand, the slope efficiency is maximum for a $100 \mu \mathrm{sec}$ pulse length at $0.34 \cdot 10^{-3}$.

Performance of the laser system when operating only on the $\mathrm{Ho}^{5} \mathrm{I}_{7}$ to ${ }^{5} \mathrm{I}_{8}$ transition was characterized using a variety of output mirror reflectivities. Using a $500 \mu \mathrm{sec}$ pump pulse and a resonator comprised of a flat output mirror and a $2.0 \mathrm{~m}$ radius of curvature high reflecting mirror, the laser output energy was measured as a function of the pump energy. For each mirror, the data was curve fit to obtain a threshold and a slope efficiency, with results of the curve fitting procedure appearing in Fig. 4. Threshold increases nearly linearly with the negative of the logarithm of the output mirror reflectivity. On the other hand, slope efficiency peaks at 0.0071 when using an output mirror reflectivity of 0.8 . A minimal decrease in the slope efficiency at the lower output mirror reflectivity indicates that up conversion losses in Ho:Tm:LuAG are not severe.

Q-switched operation was obtained on the $\mathrm{Ho}^{5} \mathrm{I}_{7}$ to ${ }^{5} \mathrm{I}_{8}$ transition. An acousto-optic modulator was used with a 0.80 reflecting mirror to effect $Q-$ switching. A single pulse could be obtained by turning the radio frequency power off for about $10 \mu \mathrm{sec}$, thus allowing a single Q-switched pulse to evolve. By then turning it back on until the population

\section{Ho:Tm:Er:LuAG Performance at 2.7 Micrometers}

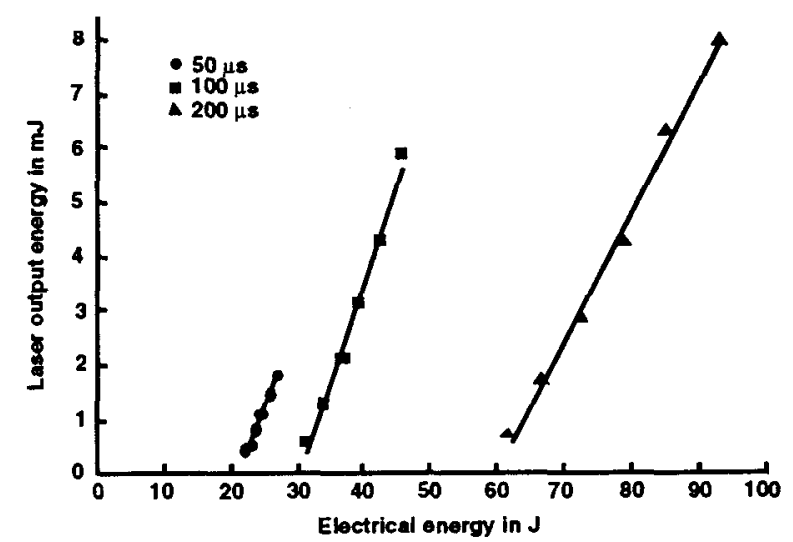

Figure 3. Laser output power at $2.7 \mu \mathrm{m}$ versus electrical energy. Three different pump pulse lengths are used. 
Threshold and Slope Efficiency of Ho:Tm:Er:LuAG 4.0 by $80 \mathrm{~mm}$ laser rod

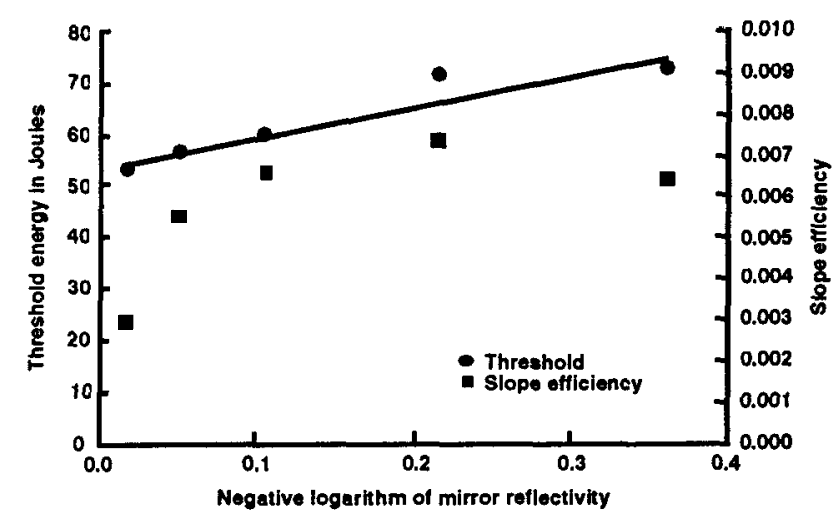

Figure 4. Threshold and slope efficiency at $2.1 \mu \mathrm{m}$ versus the negative logarithm of the output mirror reflectivity.

inversion decayed, no more Q-switched pulses were allowed to evolve. With the acousto-optic modulator inserted into the resonator, the slope efficiency decreases to 0.0051 . If the acousto-optic modulator is activated to produce a single pulse, the slope efficiency decreased to 0.0013 , indicating that a large fraction of the pump energy probably remains in the $\mathrm{Tm}{ }^{3} \mathrm{~F}_{4}$ manifold, and to some
Q-Switched Performance of Ho:Tm:Er:LuAG

4.0 by $80 \mathrm{~mm}$ lasor rod

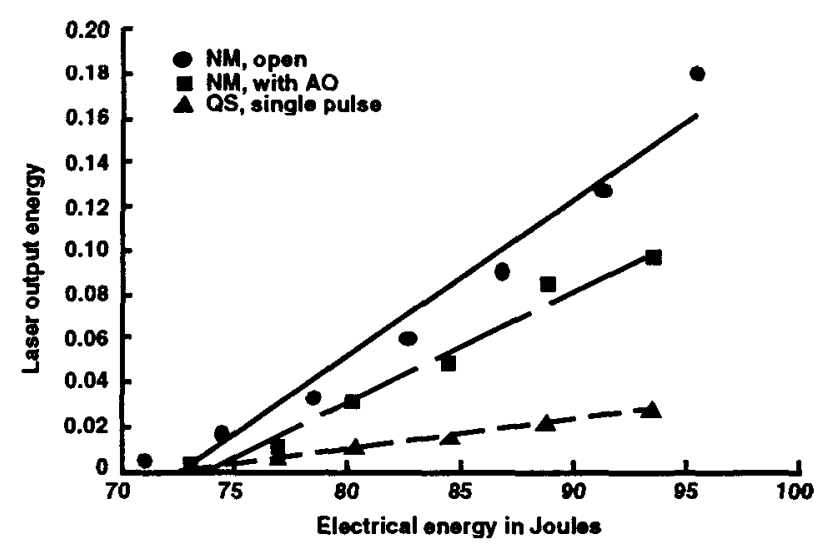

Figure 5. Normal mode and Q-switched laser output energy at $2.1 \mu \mathrm{m}$ versus pump energy. A $500 \mu \mathrm{sec}$ pump pulse length is used.

extent, the $\operatorname{Er}{ }^{4} I_{13 / 2}$ manifold. Again, a significant improvement in the performance is expected if some simple improvements in the system are implemented, such as matching the length of the laser rod to the arclength of the flashlamp and using mirrors coated especially for two wavelength operation. 